ich erlaube mir daher um so mehr, die Beobachtung seiner Minima den Astronomen recht sehr zu empfehlen. Ich bemerke dabei, dass die Beobachtung nehrere Stunden nach dem Minimum fortgesetzt werden muss, um ein zuverlässiges Resultat zn erlangen, da die Zunahme des Lichtes anfangs sebr langsam ist

Vor Kurzem hat Herr Dr. Krüger bei Gelegenheit unserer Durchmusterung auch wieder einen Stern mit sehr

Bonn 1859 Dec. 15. starker Eigenhewegung gefunden; es ist dies der Stern Groombridge No 34; seine Bewegung ist $+3^{\prime \prime} 85$ in $\mathrm{AR}$., $+0^{\prime \prime} 40$ in Decl. und $+2^{\prime \prime} 84$ im Bogen des grössten Kreises, für einell schwachen Stern $8^{\mathrm{n}}$ allerdings sehr bedeutend.

Noch erlaube ich mir, die Astronomen auf einen intensiv rothen Stern aufmerksam zu machen; es ist dies ein Stern schwach $8^{\mathrm{m}}$, dessen Position für 1855 ist $0^{\mathrm{h}} 12^{\mathrm{m}} 16^{\mathrm{g}}+43^{\circ} 54^{\prime}$, und den Krüger gleichfalls aufgefunden hat.

Fr. Argelander.

Ephemeris of the Variable Stars for the Year 1860.

By Norman Pogson, Director of the Hartwell Observatory.

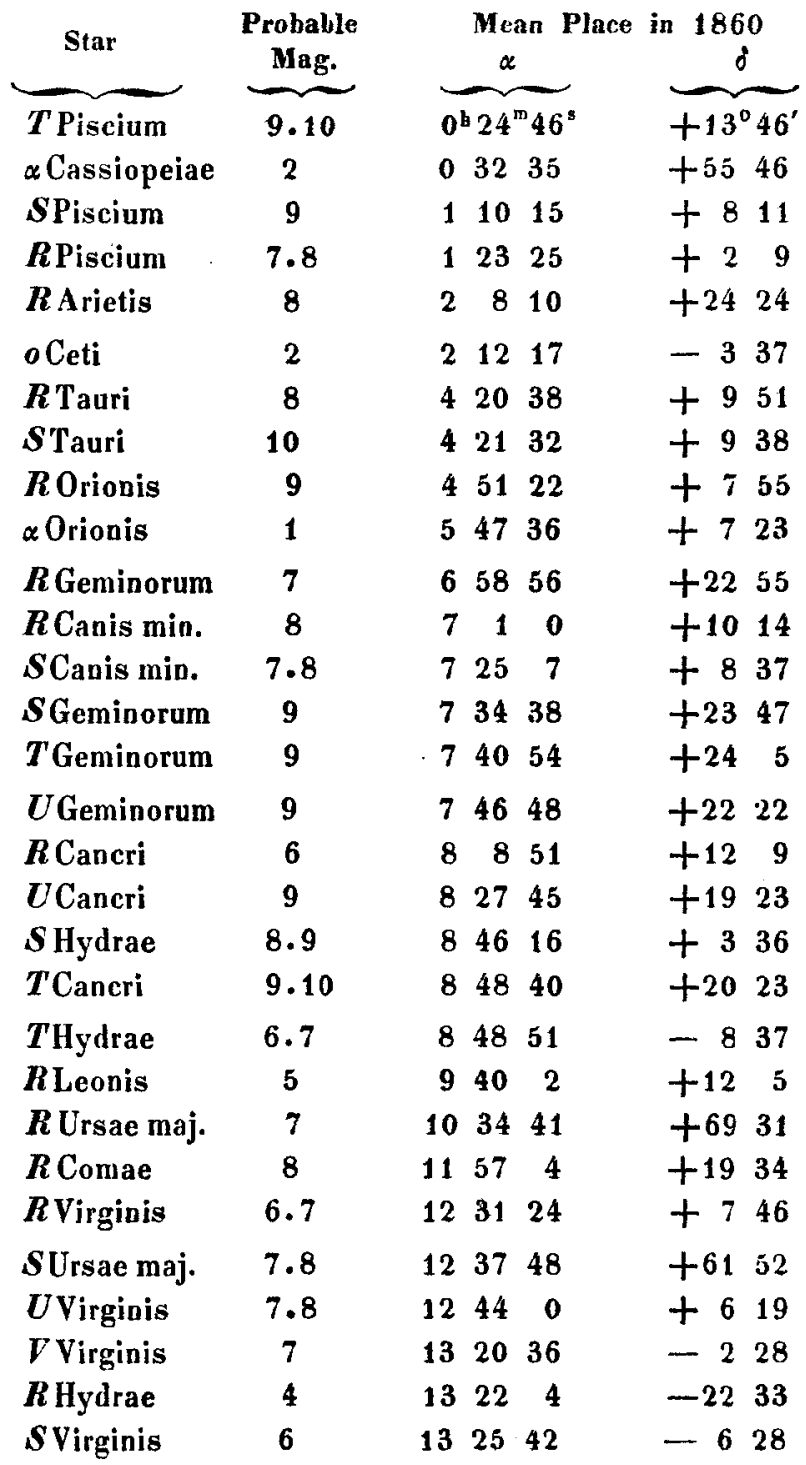

Times of Maxiun

May 7 , Sept. 27.

Jan. 19, April 8, June 26, Sept. 14, Nov. 31

June?

Early in June

Jan. 16, July 21

Sept. 6,5

July 23

Febr. 17

May 26

June and December

Jan. 15

April?

August 22

March 15 and 1861 Jan. 3

October 13

March 13, June 18, Sept. 23, Dec. 29. Irregular

April 8

October 29

August 26 so not observable

Uncertain. Minimum July 1 so not observable

July 18

Febr. 12 and 1861 Jan. 2. Minimum (10) Aug. 18

Sept. 2 Minimum (13) May 12

October?

Jan. 5,6, May 29,9, Oct. 22,2

June 2. Minima (12) Febr. 25, Oct. 7

April 8, Nov. 6

Possibly in March and November

August 31. Irregular

April 25. Minimum (11) Febr. 21
Anthority

S.

A.

B.

A.

W.

W.

W.

$\boldsymbol{P}$.

W.

P.

P.

$\boldsymbol{P}$.

A.

W.

W.

W.

W.

$\boldsymbol{P}$.

P.

W.

$\boldsymbol{A}$.

P.

W.

P.

B.

P. 


\begin{tabular}{|c|c|c|c|c|}
\hline Star & $\begin{array}{c}\text { Probable } \\
\text { Mag. }\end{array}$ & $\begin{array}{c}\text { Mea } \\
\alpha\end{array}$ & an Place & in $\begin{array}{r}\mathbf{1 8 6 0} \\
\delta\end{array}$ \\
\hline$R$ Bootis & 8 & $14^{\mathrm{h}} 31^{\mathrm{m}}$ & $1^{s}$ & $+27^{\circ} 21^{\prime}$ \\
\hline S Serpentis & 8 & 1516 & 7 & +1449 \\
\hline $\boldsymbol{R}$ Coronae & 6 & 1542 & 49 & +2835 \\
\hline $\boldsymbol{R}$ Serpentis & 6.7 & 1544 & 15 & +1534 \\
\hline $\boldsymbol{R}$ Herculis & 8.9 & 1559 & 38 & +1845 \\
\hline SScorpii & 9.10 & 169 & 17 & -2233 \\
\hline$R$ Scorpii & 9 & 169 & 19 & -2236 \\
\hline SOphiuchi & 9 & 1626 & 12 & -1652 \\
\hline SHerculis & 7.8 & 1645 & 32 & +1511 \\
\hline$R O p b i u c h i$ & 7.8 & 1659 & 44 & -1554 \\
\hline$\alpha$ Herculis & 3 & 178 & 16 & +1433 \\
\hline$T$ Herculis & 8 & 183 & 48 & +310 \\
\hline$R$ Scuti & 5 & 1840 & 1 & -550 \\
\hline 13 Lyrae & 4 & 1851 & 4 & +4346 \\
\hline $\boldsymbol{R}$ Aquilae & 6.7 & 1859 & 38 & +81 \\
\hline $\boldsymbol{R}$ Sagittarii & 8 & 198 & 28 & -1933 \\
\hline$R$ Cygni & 8 & 1933 & 4 & +4953 \\
\hline$x^{C y g n i}$ & $\mathbf{5}$ & 1945 & 11 & +3233 \\
\hline$U$ Capricorni & 10.11 & 2040 & 22 & -1518 \\
\hline$R$ Vulpeculae & 8 & 2058 & 10 & +2316 \\
\hline$T$ Capricorni & 9 & 2114 & $17 ?$ & -1545 \\
\hline$\beta$ Pegasi & 2 & 2257 & 0 & +2719 \\
\hline $\boldsymbol{R}$ Pegasi & 8.9 & 2259 & 37 & +946 \\
\hline$R$ Aquarii & 7 & 2337 & 15 & -163 \\
\hline$B$ Cassiopeiae & 6 & 2351 & 18 & +5037 \\
\hline
\end{tabular}

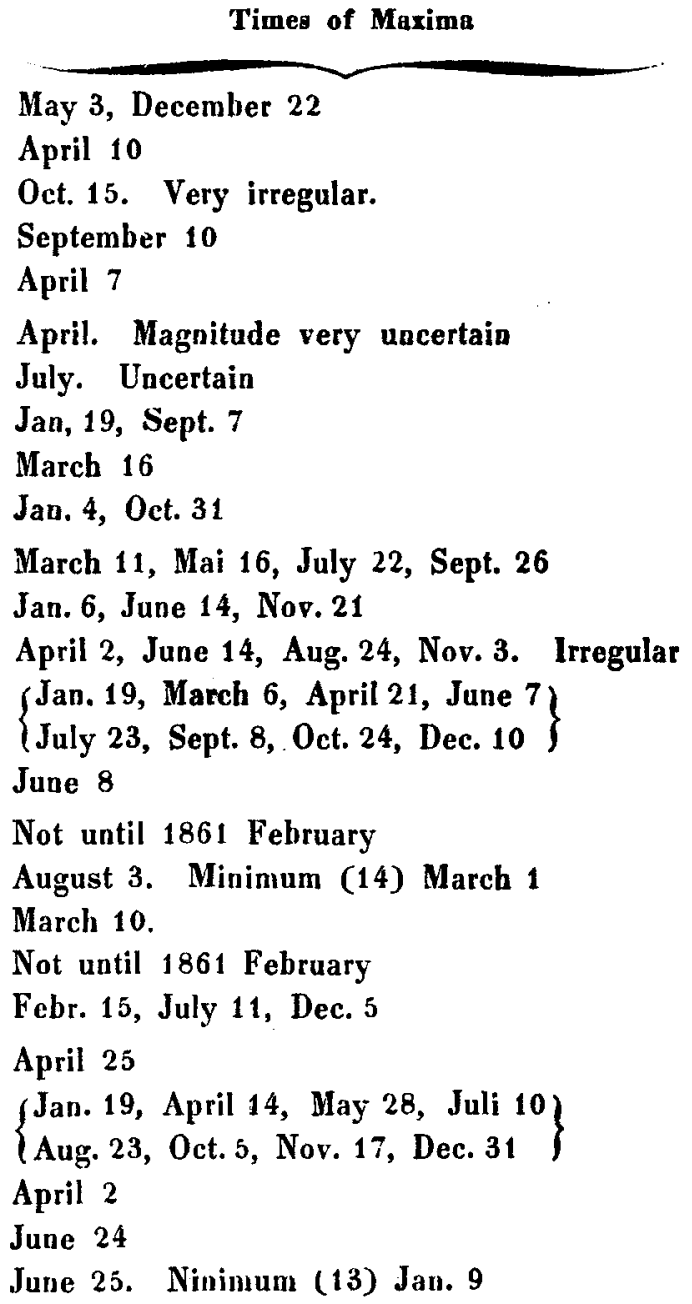

\begin{tabular}{c} 
Authority \\
\hline $\boldsymbol{W}$. \\
$A$. \\
$\boldsymbol{B}$. \\
$\boldsymbol{A}$. \\
$\boldsymbol{B}$. \\
$\boldsymbol{P}$. \\
$\boldsymbol{P}$. \\
$\boldsymbol{P}$. \\
$\boldsymbol{B}$. \\
$\boldsymbol{P}$. \\
$\boldsymbol{A}$. \\
$\boldsymbol{K}$. \\
$\boldsymbol{A}$. \\
$\boldsymbol{B}$. \\
$\boldsymbol{W}$. \\
$\boldsymbol{P}$. \\
$\boldsymbol{P}$. \\
$\boldsymbol{A}$. \\
$\boldsymbol{P}$. \\
$\boldsymbol{W}$. \\
$\boldsymbol{A}$. \\
$\boldsymbol{A}$. \\
$\boldsymbol{A}$. \\
$\boldsymbol{P}$.
\end{tabular}

Minima of the Short Period Variable Stars during 1860 in Greenwieh Mean Time and for the Earths Mean Distance from each Star.

$$
\text { A } 1 \mathrm{~g} \text { o } 1 \text {. }
$$

Maximum 2,3. Minimum 4,0. Variation completed in seven bours.

\begin{tabular}{|c|c|c|}
\hline Jan. & 2 & $16^{\mathrm{b}} 35^{\mathrm{m}}$ \\
\hline & 5 & 1324 \\
\hline & 8 & 1013 \\
\hline & 11 & $\begin{array}{ll}7 & 2\end{array}$ \\
\hline & 22 & 1817 \\
\hline & 25 & 156 \\
\hline & 28 & 1155 \\
\hline & 31 & 844 \\
\hline Febr. 1 & 14 & 1648 \\
\hline & 17 & 1337 \\
\hline & 20 & 1026 \\
\hline & 23 & 715 \\
\hline March & & 1519 \\
\hline & 11 & 128 \\
\hline & 14 & 857 \\
\hline & 31 & 1350 \\
\hline
\end{tabular}

\begin{tabular}{|c|c|c|}
\hline April & 3 & $10^{\mathrm{h}} 39^{\mathrm{m}}$ \\
\hline & 20 & $15 \quad 32$ \\
\hline & 23 & 1221 \\
\hline & 26 & 910 \\
\hline May & 13 & 143 \\
\hline & 16 & 1052 \\
\hline June & $\mathbf{5}$ & 1234 \\
\hline & 8 & 923 \\
\hline & 11 & $14 \quad 16$ \\
\hline & 28 & 115 \\
\hline July & 18 & .1247 \\
\hline & 21 & 936 \\
\hline Aug. & 7 & 1429 \\
\hline & 10 & 1118 \\
\hline & 30 & 130 \\
\hline Sept. & 2 & 949 \\
\hline
\end{tabular}

$\begin{array}{lrrr}\text { Sept. } & 19 & 14^{\mathrm{b}} & 42^{\mathrm{m}} \\ & 22 & 11 & \mathbf{3 1} \\ & 25 & 8 & 20 \\ \text { Oct. } & 9 & 16 & 24 \\ & 12 & 13 & 13 \\ & 15 & 10 & 2 \\ & 18 & 6 & 51 \\ \text { Nov. } & 1 & 14 & 55 \\ & 4 & 11 & 44 \\ 7 & 8 & 33\end{array}$
Nov. $10 \quad 5^{\text {h }} 22^{\mathrm{m}}$
Dec. $11 \quad 18 \quad 19$ $\begin{array}{lll}14 & 15 & 8\end{array}$

\section{$\lambda \mathbf{T}$ a u $\mathbf{r}$.}

Maximum 4,0. Minimum 4,5. Variation rapid.

\begin{tabular}{lrrrrrr} 
Jan. 31 & $13^{\mathrm{h}}$ & $8^{\mathrm{m}}$ & Febr. 16 & \multicolumn{2}{c}{$8^{\mathrm{h}} 7^{\mathrm{m}}$} \\
Febr. 4 & 12 & 0 & & 20 & 7 & 29 \\
8 & 19 & 52 & Sept. 28 & 16 & 12 \\
12 & 9 & 44 & Oct. 2 & 15 & 4
\end{tabular}


C $G$ e m i n o r u m.

Maximum 3,8. Minimum 4,5. Increasing $5^{d} 6^{b}$.

Jan. $30 \quad 5^{\mathrm{h}} 48^{\mathrm{mi}} \quad$ April $20 \quad 12^{\mathrm{h}} 12^{\mathrm{h}}$

Fehr. $9 \quad 936 \quad$ Oct. $30 \quad 1224$

$19 \quad 1324 \quad$ Dec. $20 \quad 724$

April $10 \quad 8 \quad 24 \quad 30 \quad 11 \quad 12$

S C a ncri. $\quad x=8^{\mathrm{h}} 35^{\mathrm{m}} 56^{\mathrm{s}} \quad d=+19^{\circ} 32^{\prime}$.

Maximum 8,0. Minimum 10,5. Variation rapid.

Jan. $2 \quad 13^{\mathrm{h}} 5^{\mathrm{m}} \quad$ Sep $\quad 14 \quad 14^{\mathrm{h}} 41^{\mathrm{m}}$

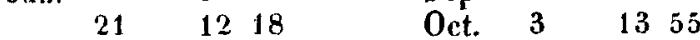

Febr. $9 \quad 11 \quad 32 \quad 22 \quad 13 \quad 9$

$\begin{array}{llllll}28 & 10 & 46 & \text { Nov. } 10 & 12 & 23\end{array}$

$\begin{array}{llllll}\text { March } 18 & 10 & 0 & 29 & 11 & 37\end{array}$

April $6 \quad 9 \quad 14 \quad$ Dec. $18 \quad 1051$

$\beta$ L y a e.

Principal Minimum $(4,3)$. Fîrst Maximum $(3,4) . \quad 3^{\mathrm{d}} 2^{\mathrm{h}}$ after. Second Min. $(3,8) 6^{\text {d }} 9^{\mathrm{h}}$ after. Second Max. $(3,4) 9^{\mathrm{d}} 10^{\mathrm{h}}$ after.

\begin{tabular}{|c|c|c|c|}
\hline Jan. 2 & $5^{\mathrm{h}} 11^{\mathrm{m}}$ & Sept. 14 & $12^{h} 44^{n}$ \\
\hline April 1 & 1542 & $\begin{array}{l}-27 \\
\end{array}$ & 1030 \\
\hline & 1327 & Oct. & 815 \\
\hline May & $11 \quad 12$ & 23 & 0 \\
\hline Sept. & $\begin{array}{r}858 \\
1459\end{array}$ & Dec. & 1846 \\
\hline
\end{tabular}

$$
\eta \text { A q u i l a e. }
$$

Maximum 3,6. Minimum 4,4. Increasing $2^{\mathrm{d}} 9^{\mathrm{h}}$.

$\begin{array}{rrrrrrr}\text { Febr. 16 } & 18^{\mathrm{b}} 9^{\mathrm{m}} & \text { Aug. } 21 & 8^{\mathrm{h}} & 1^{\mathrm{m}} \\ \text { March23 } & 15 & 20 & & 28 & 12 & 29 \\ \text { April } 28 & 12 & 30 & \text { Oct. } & 3 & 9 & 40 \\ \text { June 10 } & 13 & 55 & \text { Nov. } & 8 & 6 & 50 \\ \text { July } 16 & 11 & 5 & \text { Dec. } 14 & 4 & 0 \\ 23 & 15 & 19 & & & & \end{array}$

$$
\text { d C e p h e i. }
$$

Maximum 3,7. Minimum 4,8. Increasing $1^{\mathrm{d}} 14^{\mathrm{h}}$.

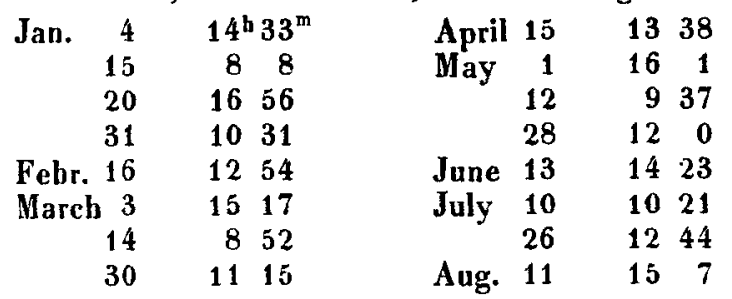

$\begin{array}{lrrrrrrr}\text { Aug. } & 22 & 8 & 42 & \text { Nov. } & 11 & 50 \\ \text { Sept. } & 7 & 11 & 5 & & 16 & 5 & 25 \\ & 23 & 13 & 28 & & 21 & 14 & 13 \\ \text { Oct. } & 4 & 7 & 4 & \text { Dec. } & 2 & 7 & 48 \\ & 9 & 15 & 51 & & 7 & 16 & 31 \\ & 20 & 9 . & 27 & & 18 & 10 & 11\end{array}$

The minima of $\lambda$ Tauri were obligingly furnished by M. Baxendell. These of all the other short period Variables depend upon Prof. Aryelander's last published elements of variation.

The initials in the last columne of the first table stand lor the names of Argelander, Aumer's, Baxendell, Kriiger, Pogson, Schönfeld and Winnecke, respectively. The times of maxima of the four stars without any letter annexed are merely probable conjectures; too uncertain to be given as upon any authority whatever! Dr. Winnecke's valuable paper in Astr. Nachr. 1224 and M. Baxendell's friendly communications, together with a preparatory discussion of several years observations of the stars given on my own responsibility, have enabled me to offer a far more trustworthy ephemeris than on any previous occasion; though nearly forty stars are still omitted fiom the want of statisfactory epochs and periods.

For $\boldsymbol{R}$ Arietis Winnecke gives the maxima Jan. 15, July 19

For $U$ Geninorum $==\quad=$ March 11, Sept.21,

For $S$ Virginis $=\quad=\quad=$ April 21 . Dec. 27.

$V$ Virginis is an interesting star discovered by Herr Hermann Goldschmidt in 1857. $S$ and $S$ Scorpii have unfortunately been incorrectly given in position hitherto. The place of the former although a mere estimation is fairly approximate; that of its neighbor $R$ is from observation.

Dr. Winnecke gives the Declination of $\boldsymbol{s}$ Serpentis $+14^{\circ} 29^{\prime}$ instead of $+14^{\circ} 49^{\prime}$ and $I$ am unable to say which is right, not having yed observed the star myself.

The Right Ascension of $T$ Capricorni is also uncertain by several seconds.

In Astr. Nachr. 1183 Herr Auwers gives his independent discovery of change in J Orionis. Sir John Herschel however positively declared this star to be variable imany years back (see R. A. S. Monthly Notices, Vol. IV., p. 196), though 1 am not aware that any particulars of its variation were ever published, and even his assertion appears to have been overlooked.

Hartwell Observatory 1859 Dec. 24.

N. R. Pogson.

\section{Literarische Anzeige.}

Herr General von Schubert theilte mir mit, dass er bei der Benutzung der Besselschen Gradmessung in der Abhandlung „Essai d'une détermination de la véritable figure de la Terre," welche in $N_{*}^{o} 1201$ dieser Blätter angezeigt ist, einen Rechnungsfehler begangen habe. Um diesen Irrthum zu berichtigen und zugleich die Resultate der grossen Englischen Vermessung, wie sie in dem kürzlich erschienenen „Ordnance trigonometrical survey" mitgetheilt sind, zu vergleichen, bat Herr von Schubert den letzten Bogen jener Abhandlung umdrucken lassen.

Die in 1201 der A. N. abgedruckten Elemente des dreiachsigen Ellipsoids sind ungeändert geblieben und stellen die Anıplituden der verschiedenen Gradmessungen folgendermaassen dar:

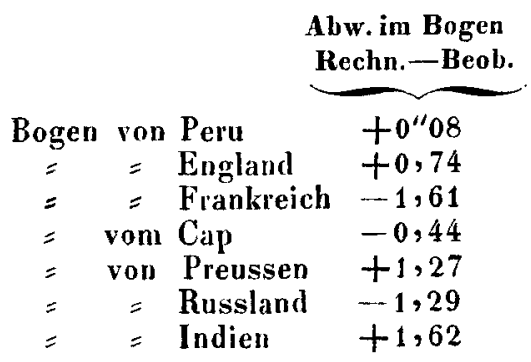

P. 